\title{
Performance Analysis for the Magnetically Coupled Resonant Wireless Energy Transmission System
}

\author{
Jinguo Liu $\mathbb{D},{ }^{1}$ Xuebin Zhang, ${ }^{2}$ Jiahui Yu, ${ }^{3}$ Zhenyao Xu, ${ }^{1}$ and Zhaojie Ju $\mathbb{D}^{1,3}$ \\ ${ }^{1}$ State Key Laboratory of Robotics, Shenyang Institute of Automation, Chinese Academy of Sciences, Shenyang 110016, China \\ ${ }^{2}$ Beijing Institute of Structure and Environment Engineering, Beijing 100076, China \\ ${ }^{3}$ School of Computing, University of Portsmouth, Portsmouth PO1 3HE, UK \\ Correspondence should be addressed to Zhaojie Ju; zhaojie.ju@port.ac.uk
}

Received 19 July 2019; Accepted 10 October 2019; Published 11 November 2019

Guest Editor: Jianwu Zeng

Copyright (c) 2019 Jinguo Liu et al. This is an open access article distributed under the Creative Commons Attribution License, which permits unrestricted use, distribution, and reproduction in any medium, provided the original work is properly cited.

\begin{abstract}
As a new wireless energy transmission technology, magnetically coupled resonant wireless energy transmission system (MCRETS) is not easily affected by obstacles in the transmission process, and the transmission distance is relatively far. However, how to balance the relationship between transmission efficiency and power to achieve optimal performance is still a huge challenge. In addition, few studies have theoretically investigated the factors affecting the wireless energy transmission system to obtain an optimal solution. Here, through unprecedented theoretical analysis, we find the exact parameters of system optimization and verify them by simulation and experiments. First, the optimal topology of MCRETS is obtained through theoretical analysis and comparison of topologies. Second, to improve the transmission performance of MCRETS, its impact factors, including transmission distance, resonant frequency, relay coil, and relative position of launch and receiving coils, are analyzed in detail to get accurate parameters. Furthermore, based on the analysis, we propose an unprecedented concept for balancing optimal efficiency and power, which is named the power product. Finally, the effectiveness of the proposed method is verified through analysis and experimental results. These findings shed light on the relationship between efficiency and power and provide a comprehensive theoretical basis for subsequent research.
\end{abstract}

\section{Introduction}

With the increase in the number of traditional receiving terminals of cable power supply, the drawbacks, such as socket exposure, plugs prone to sparks, and other issues, become increasingly evident. To solve these problems, wireless power transfer (WPT) is utilized and it achieves wireless transmission through mutual coupling among physical fields. This technology has improved both equipment safety and functionality and has currently gained attention in the field of electrical research [1], especially in the current hotspots of electric vehicles [2] and wireless sensor networks [3]. At present, noncontact WPT can be divided into three forms, namely, electromagnetic induction, microwave, and magnetic resonant coupling. Electromagnetic induction and microwave development are relatively mature and are mainly used in maglev trains [4] and bodies of microcamera power supplies [5]. In a magnetically coupled resonant wireless energy transmission system (MCRETS), the magnetic fields serve as transmission media and create energy for wireless transmission through their resonance [6-8]. This technology has high transmission efficiency, can transmit a large amount of power, and is not susceptible to certain metals found in transmission channels [9]. Compared with electromagnetic induction and microwave transmission techniques, MCRETS is essentially different in the following ways: first, magnetic resonant coupling has greater transmission distance limit that is far beyond the limit of electromagnetic induction [10], and moreover, its transmission distance can be further increased even without regard to the impact of other factors, such as the loss of energy in the air, the influence of magnetic material, and other devices emitting electromagnetic interference in the transmission space; second, compared with the microwave WPT, transmission power of magnetic resonant coupling is greater. Overall, MCRETS is new and more widely used [11], 
given that it meets the requirements for electromagnetic compatibility and has a high application value and broad applications in medical implantation machinery [12], robots [13], wireless sensors [5], and consumer appliances [14].

Researchers have made numerous breakthroughs in the study on MCRETS [15]. For example, Soljacic used the principle of magnetic resonant coupling to light a $60 \mathrm{~W}$ bulb from a distance of $2.16 \mathrm{~m}$. The transmission efficiency of the bulb reached $40 \%$. The energy transfer mechanism between the two resonators was analyzed by using the coupling mode theory. In [16], the coupling mode theory was also utilized in analyzing the energy transfer among three resonant coils and the influence of relay coil. In [17], a physical model of the equivalent circuit of transmitting and receiving coils was established, a four-coil wireless energy transmission model for medical implants was optimized, and the main factors that influenced the efficiency of the system were theoretically analyzed. In [18], a two-layer nested optimization method based on the differential evolution algorithm was proposed, and the maximum transmission efficiency of the transmission system was theoretically studied and verified through the finite element method. Another study introduced the working principle of MCRETS, and the impact factors of the transmission performance (e.g., transmission distance) of the system were theoretically analyzed [19]. In [20], physical analysis of the wireless energy transmission system with a magnetic field repeater was performed, and the influence of the magnetic relay on transmission performance of the system was theoretically analyzed. In [21], the physical structure and working principle of the low-power wireless energy transmission system applied to medical devices were theoretically analyzed. Besides, research on high-power applications has made some progress. In [22], researchers studied the optimization of the coil; in [23], the magnetic coupling has been improved, through using the method of adding ferrite core materials. However, most of the designs in previous studies have modeled single transmissions, maximum power transmission, or transmission at highest efficiency. No design based on dual transmission indices, such as those that modeled simultaneous transmission or maximum transmission efficiency, is available. In addition, few papers have theoretically analyzed the influence of multiple factors on transmission performance because the bulk of the few existing studies contends with single impact factors. Moreover, considerably few experiments have performed to verify theories on optimization of transmission, such as those that posit low transmission efficiency occurs when wireless energy transmission system meets the maximum transmission power.

To address the above issues, a complete modeling and analysis of the wireless energy transmission system based on the transmission efficiency and power is conducted in this study. The basic structure, working principles of the wireless energy transmission system, and topology are analyzed and compared. The four impact factors of transmission performance (i.e., transmission distance, system resonance frequency, relay structure, and relative position of transmitting and receiving coils) are theoretically analyzed to derive the degree of influence of each factor. Although the size and shape of the coil have an impact on the transmission performance, the nature of such effects is the coil's own parameter settings, such as resistance, which have little effect on practical applications. Therefore, the research factors in this paper set the transmission distance, the resonant frequency, and the relative position of the transmitting and receiving coils. The main reason is that the influence of these factors is more common in practical applications. The validity of the theoretical analysis is experimentally verified. This study also creatively presents the power product parameters, which are theoretically analyzed to obtain a conductive transmission state and optimize the transmission power and transmission efficiency. This analysis can provide theories and solutions for the design and optimization of MCRETSs. The main contributions of this paper are summarized as follows:

(1) We present the complete modeling and analysis of the wireless energy transmission system based on the transmission efficiency and transmission power of two transmission indicators, analysis of its basic structure and working principle, and analysis and comparison for the optimal topology.

(2) The influencing factors of this paper are different from the traditional methods and previous studies. Firstly, we get the exact effect of the four influencing factors from the theoretical deduction and then verify the correctness of the theoretical deduction through experiments. In addition, we get the theoretical value of the turning point, which has a certain significance for optimizing practical problems.

(3) Comparing the factors that affect this system performance listed before, considering the problem that power and efficiency optimization cannot be both concerned in practical applications, this paper presents a novel method of optimal selection, named as power product and elaborates its derivation principle and experimental process in detail.

(4) The theoretical analysis is verified through an experiment, and power product parameters are proposed to reach an optimal transmission power and efficiency of the system, with theoretical analysis.

The remainder of this paper is as follows: Section 2 reviews the related work of the wireless energy system. In Section 3, we introduce the process of the proposed method. The validation of the proposed method using finite element simulation is presented in Section 4. In Section 5, we report various experimental results as well as the comparison with the state-of-the-art methods. Finally, Section 6 concludes this paper with the summary and discussion.

\section{Structure and Working Principle of the Wireless Energy Transmission System}

In a vibrating system, strong coupling states often exist among several parts with the same intrinsic frequency [24]. The magnetically coupled resonance is a strong coupling 
method of adjacent magnetic fields, wherein loss of energy in the transmission is minimized even with high transmission efficiency. It is not easily affected by metal barriers and other disruptive materials found in the transmission channel, and it can generate nondirectional transmission.

The core structure of MCRETS comprises two or more designed resonant coils: a matching module for supplying high-frequency energy that is equal to the intrinsic frequency of the resonant coil and supporting modules that receive high-frequency energy and change in the form of energy required. Figure 1 shows a typical midrange block diagram of MCRETS, which mainly includes a frequency AC power supply, a rectifier filter module, a RF amplifier, an impedance matching and regulation control circuit, transmitting and receiving coils, a rectifier, and a load. After the RF amplifier receives transmission, the receiver coil resonates a current, and the wireless energy transmission system achieves the maximum efficiency.

MCRETS has an external capacitor resonant compensation for each loop. External capacitors can be divided into series and parallel compensations by the capacitive access circuit. The compensation structure is discussed separately for the primary and secondary circuits. Resonant compensation structures can be divided into series-series (SS), series-parallel (SP), parallel-series (PS), and parallel-parallel (PP) [25]. The four circuit compensation structures are shown in Figure 2.

In Figure 2, the subscript $P$ denotes the primary circuit, $U$ is the primary-induced voltage source, IP is the loop current, LP and RP are the equivalent circuit parameters of the coil, and CP is the tuning capacitance in the primary circuit. Subscript $S$ represents the secondary circuit, RL is the load, IS is the loop current, LS and RS are coil equivalent circuit parameters in the secondary circuit, CS is the tuning capacitor in the secondary circuit, and $M$ is the mutual inductance between the two circuits.

If the frequency of the transmission system is $f$, the equations of the primary and secondary circuits are as follows:

$$
\left\{\begin{array}{l}
\omega=2 \pi f, \\
Z_{\mathrm{P}} \dot{I}_{\mathrm{P}}-j \omega M \dot{I}_{\mathrm{S}}=U, \\
-j \omega M \dot{I}_{\mathrm{P}}+Z_{\mathrm{S}} \dot{I}_{\mathrm{S}}=0,
\end{array}\right.
$$

where ZP and ZS are the impedances of the primary and secondary circuits. The currents of the primary and secondary circuits, IP and IS, can be obtained from (1):

$$
\left\{\begin{array}{l}
\dot{I}_{\mathrm{P}}=\frac{U}{Z_{\mathrm{P}}+(\omega M)^{2} / Z_{\mathrm{S}}}, \\
\dot{I}_{\mathrm{S}}=\frac{j \omega M U / Z_{\mathrm{P}}}{Z_{\mathrm{S}}+(\omega M)^{2} / Z_{\mathrm{P}}},
\end{array}\right.
$$

where $Z_{\mathrm{PS}}+(\omega M)^{2} / Z_{\mathrm{S}}$ is the response impedance of the secondary circuit to the primary circuit and $Z_{\mathrm{SP}}+(\omega M)^{2} / Z_{\mathrm{P}}$ is the response impedance of the primary circuit to the secondary circuit. When $\omega_{0}=1 / \sqrt{L_{S} C_{S}}$, the resonant frequency of each coil in a transmission system is consistent

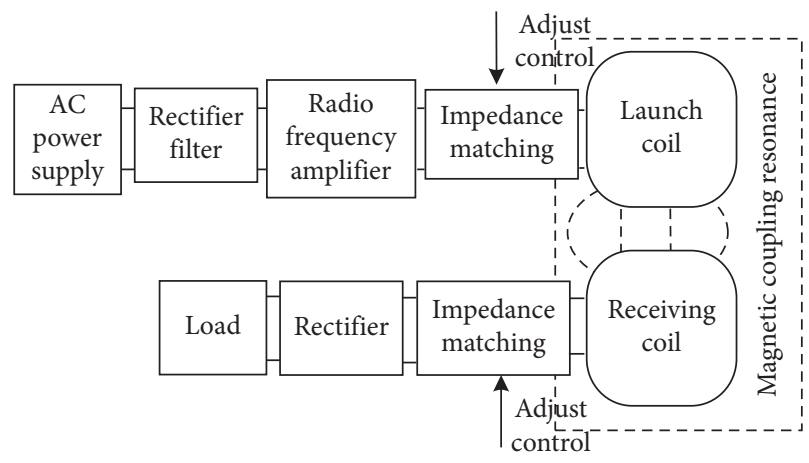

FIGURE 1: Block diagram of a medium-distance MCRETS.

with the resonant frequency of the excitation source, and the system transmission efficiency is the highest. Moreover, the currents in the two loops are affected by the opposing loops in (2).

When the resonant compensation circuit is a series structure, impedance ZPS is purely resistant to the resonant frequency, whereas the two coexist in a parallel structure. When the system has a SS compensation structure, the external resonant capacitor is unaffected by the impedance of the secondary loop, the transmission distance, or load characteristics, and the resonant frequency does not affect the choice of the resonant capacitor, so the system design optimization is more convenient. Thus, the topology of the external resonant capacitance has a SS structure.

\section{Theoretical Analysis of the Transmission Performance of MCRETS}

In traditional methods, most of the previous studies are directly through simulation to get the optimal parameters of each influencing factor, so only the optimal parameters range can be obtained. This paper is different from traditional methods. Firstly, starting with the theoretical model, the exact value of the optimal parameters is deduced by formula, and then the deduced results are verified by simulation and experiment. In this way, we can find the essence of each factor and provide a theoretical basis for future research.

In this chapter, we theoretically analyze the influence factors of transmission performance (transmission distance, system resonance frequency, relay structure, relative position of transmitting, and receiving coils) and get the influence of each factor.

\subsection{Influence of Transmission Distance on the Transmission} Performance of MCRETS. The results and discussion may be presented separately, or in one combined section, and may optionally be divided into headed subsections. The transmission efficiency of MCRETS is defined as the ratio of the power at the load side to the power transmitted by the transmitter. The transmission performance of MCRETS is susceptible to the four factors, namely, transmission distance, resonant frequency, relay coil, and relative position of 


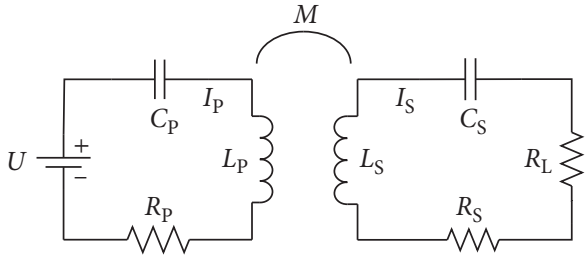

(a)

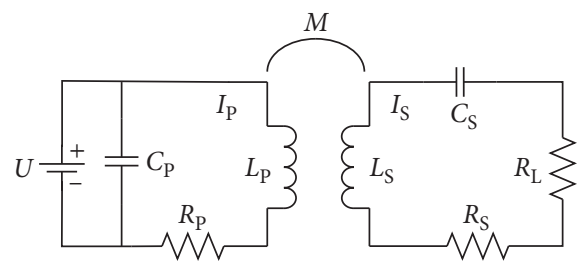

(c)

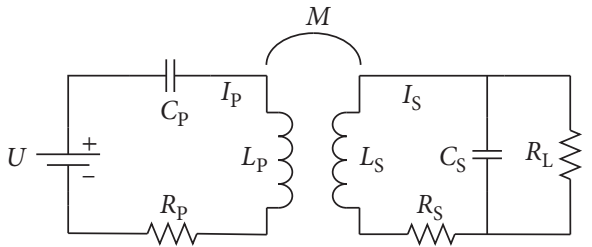

(b)

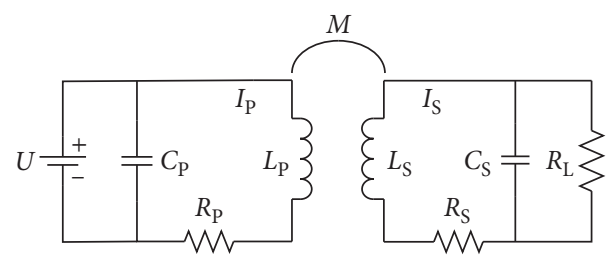

(d)

FIgURE 2: Four basic resonant compensation structure circuits: (a) SS mode; (b) SP mode; (c) PS mode; (d) PP mode.

transmitting and receiving coils. According to the mechanism of magnetic coupling of resonant wireless energy transmission, resonant frequency is proportional to and can directly affect the transmission distance. In principle, the energy efficiency of short-range applications can be quite high, but it will drop rapidly as the transmission distance increases [26, 27]. In this study, the impact factor of transmission distance is theoretically analyzed. The diagram of the reception and equivalent circuits of MCRETS is shown in Figure 3.

The equivalent impedance of the transmitting and receiving circuits is

$$
\left\{\begin{array}{l}
Z_{\mathrm{P}}=\frac{R_{\mathrm{P}}+1}{j \omega C_{\mathrm{P}}+j \omega L_{\mathrm{P}}}, \\
Z_{\mathrm{S}}=\frac{R_{\mathrm{S}}+1}{j \omega C_{\mathrm{S}}+j \omega L_{\mathrm{S}}},
\end{array}\right.
$$

when the resonant frequency of the system $\omega=1 / \sqrt{C_{\mathrm{P}} L_{\mathrm{P}}}=1 / \sqrt{C_{\mathrm{S}} L_{\mathrm{S}}}$, the equivalent impedance of the series resonant of the transmitting circuit is approximately 0 , and the equivalent impedance of the parallel resonator is infinite. In the coil-resonant coupling circuits shown in Figure 3, the SS topology can be easily analyzed by systematically integrating it into a resonant system that only contains transmitting and receiving coils. The following formula can be obtained from Figure 3:

$$
\left\{\begin{array}{l}
Z_{\mathrm{P}} \dot{I}_{\mathrm{P}}-j \omega M \dot{I}_{\mathrm{S}}=\dot{U}_{i}, \\
-j \omega M \dot{I}_{\mathrm{P}}+Z_{\mathrm{S}} \dot{I}_{\mathrm{S}}=0 .
\end{array}\right.
$$

Then,

$$
\left\{\begin{array}{l}
Z_{\mathrm{P}}=\frac{R_{\mathrm{P}}+1}{j \omega C_{\mathrm{P}}+j \omega L_{\mathrm{P}}}, \\
Z_{\mathrm{S}}=\frac{R_{\mathrm{S}}+1}{j \omega C_{\mathrm{S}}+j \omega L_{\mathrm{S}}} .
\end{array}\right.
$$

$\mathrm{ZP}$ and $\mathrm{ZS}$ are incorporated into (5), as follows:

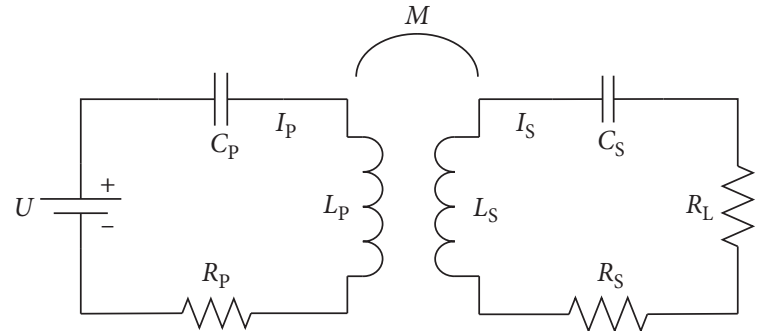

FIGURE 3: Equivalent circuit diagram of the transmitting and receiving circuits.

$$
\left\{\begin{array}{l}
\left(\frac{R_{\mathrm{P}}+1}{j \omega C_{\mathrm{P}}+j \omega L_{\mathrm{P}}}\right) \dot{I}_{\mathrm{P}}-j \omega M \dot{I}_{\mathrm{S}}=\dot{U}_{i}, \\
-j \omega M \dot{I}_{\mathrm{P}}+\left(\frac{R_{\mathrm{S}}+1}{j \omega C_{\mathrm{S}}+j \omega L_{\mathrm{S}}}\right) \dot{I}_{\mathrm{S}}=0 .
\end{array}\right.
$$

When $\omega_{0}=1 / \sqrt{C_{\mathrm{S}} L_{\mathrm{S}}}=1 / \sqrt{C_{\mathrm{P}} L_{\mathrm{P}}}$, the system reaches a resonant state. The mutual inductance expression between the two coaxially parallel hollow coils is

$$
M=\frac{\pi \mu_{0} r^{4} n^{2}}{2\left(\sqrt{2 r^{2}+D^{2}}\right)^{3}} .
$$

Coil resistance can be equivalent to $R_{\mathrm{P}}=R_{\mathrm{S}} \approx R_{0}=\sqrt{\omega \mu_{0} / 2 \sigma} n r / a$, where $\sigma$ is the conductivity, $D$ is the transmission distance, $\mu_{0}$ is the vacuum permeability, $r$ is the radius of the transmitter and receiver, and a and $n$ indicate the radius of the wire of the receiving and transmitting coils and the number of turns of the two coils, respectively.

Equation (6) is solved as follows:

$$
\left\{\begin{array}{l}
I_{\mathrm{P}}=\frac{\left(R_{0}+R_{\mathrm{L}}\right) U_{i}}{R_{0}\left(R_{0}+R_{\mathrm{L}}\right)+(\omega M)^{2}}, \\
I_{\mathrm{S}}=\frac{j \omega M U_{i}}{R_{0}\left(R_{0}+R_{\mathrm{L}}\right)+(\omega M)^{2}} .
\end{array}\right.
$$


The output power and transmission efficiency of the load can be expressed as follows:

$$
\left\{\begin{array}{l}
P_{0}=I_{\mathrm{S}}^{2} R_{\mathrm{L}}=\frac{(\omega M)^{2} U_{i} R_{\mathrm{L}}}{\left[R_{0}\left(R_{0}+R_{\mathrm{L}}\right)+(\omega M)^{2}\right]^{2}}, \\
\eta=\frac{P_{0}}{P_{i}}=\frac{I_{d}^{2} R_{\mathrm{L}}}{I_{\mathrm{P}} U_{i}}=\frac{(\omega M)^{2}}{(\omega M)^{2}+R_{0}\left(R_{0}+R_{\mathrm{L}}\right)} .
\end{array}\right.
$$

When resonant frequency $\omega$, load resistance $R_{\mathrm{L}}$, transmitting and receiving coil parameters, and coil mutual inductance $M$ can affect the transmission efficiency and output power of MCRETS, the magnitude of the mutual inductance is related to the transmitting distance. Thus, the transmission distance is related to the mutual inductance between the transmitting and receiving coils, the transmission efficiency of the wireless energy transmission system, and the output power.

From (9), the output power is derived from mutual inductance $M$; that is, when $\mathrm{d} P_{0} / \mathrm{d} M=0$, the extreme point is obtained as

$$
\left\{\begin{array}{l}
M=\frac{\sqrt{\left(R+R_{0}\right)\left(R_{0}+R_{\mathrm{L}}\right)}}{\omega}, \\
M=\frac{\pi \mu_{0} r^{4} n^{2}}{2\left(\sqrt{2 r^{2}+D^{2}}\right)^{3}} .
\end{array}\right.
$$

The extreme distance can be derived from (10), as follows:

$$
D_{0}^{2}=\sqrt[3]{\frac{\left(\omega^{2} T^{2}\right)}{\left[\left(R+R_{0}\right)\left(R_{0}+R_{\mathrm{L}}\right)\right]}}-2 r^{2} .
$$

Then, $T=\pi \mu_{0} r^{4} n^{2} / 2$.

Furthermore, when $D<D_{0}, P_{0}<P$ max; when $D<D_{0}$, $P_{0}<P$ max; and when $D=D_{0}, P_{0}=P \max$, in which the power is the largest. Thus, when the transmission distance changes, the output power of the load initially increases and then decreases with the increase in the transmission distance.

Similarly, the relationship between mutual inductance and transmission efficiency can be obtained from mutual inductance $M$ :

$$
\frac{\mathrm{d} \eta}{\mathrm{d} M}=\frac{2 \omega^{2} M\left(R+R_{0}\right)\left(R_{0}+R_{\mathrm{L}}\right)}{\left[\left(R+R_{0}\right)\left(R_{0}+R_{\mathrm{L}}\right)+(\omega M)^{2}\right]^{2}},
$$

where $\mathrm{d} \eta / \mathrm{d} M>0$ is established; that is, transmission efficiency is proportional to the size of mutual inductance because the mutual inductance and transmission distance are inversely proportional. Consequently, transmission efficiency decreases with the increase in transmission distance.

\subsection{Influence of Resonant Frequency on the Transmission} Performance of MCRETS. The resonant frequency of MCRETS is the driving signal frequency. When the driving signal frequency and the natural frequency of the transmitting and receiving coils are consistent, the system can reach a resonant state. When the output power in (9) is derived from the resonant frequency, that is, $\mathrm{d} P_{0} / \mathrm{d} \omega=0$, the extreme frequency can be derived as follows:

$$
\omega_{0}=\frac{\sqrt{\left(R+R_{0}\right)\left(R_{0}+R_{\mathrm{L}}\right)}}{M} .
$$

Based on the above analysis, when $\omega<\omega_{0}, \mathrm{~d} P_{0} / \mathrm{d} \omega>0$; when $\omega>\omega_{0}, \mathrm{~d} P_{0} / \mathrm{d} \omega<0$; and when $\omega=\omega_{0}, \mathrm{~d} P_{0} / \mathrm{d} \omega=0$. Thus, the output power of the system initially increases and then decreases with the increase of the resonant frequency. When $\omega=\omega_{0}$, the output power is the largest.

The resonant frequency is derived from the transmission efficiency, that is, $\mathrm{d} \eta / \mathrm{d} \omega=0$ :

$$
\frac{\mathrm{d} \eta}{\mathrm{d} \omega}=\frac{2 \omega M^{2}\left(R+R_{0}\right)\left(R_{0}+R_{\mathrm{L}}\right)}{\left[\left(R+R_{0}\right)\left(R_{0}+R_{\mathrm{L}}\right)+(\omega M)^{2}\right]^{2}} .
$$

In (14), $\mathrm{d} \eta / \mathrm{d} \omega>0$ is established; thus, the transmission efficiency increases with the resonant frequency. However, in an actual setup, the skin effect will increase the transmission efficiency of the system and increases in the resonant frequency tend to be stable.

3.3. Wireless Energy Transmission System with a Relay Structure. The four-coil structure of MCRETS can achieve energy in a medium-distance transmission. When the distance of wireless energy transmission exceeds the critical coupling distance and into the under-coupled area, the transmission performance of the system will decline. However, adding a relay coil between the transmitting and receiving coils will improve the transmission performance of the system. The diagram of a five-coil wireless energy transmission system is shown in Figure 4, in which the relay and resonant coils can be consistent.

In this structure, the relay coil is equivalent to the circuit, and the resonant capacitor is serially connected with the circuit. The equivalent circuit of the five-coil wireless energy transmission system is shown in Figure 5. To simplify the model, the mutual inductance between nonadjacent coils can be ignored.

The relay and transmitting coils are near with each other; thus, they can be regarded as a transmitting source with a relay coil. The relay coil itself has a high-quality factor, which can increase the resonant current and enhance the magnetic field effect. This condition allows the relay and receiving coils in the long-distance coupling resonance to increase the transmission distance and enhance the transmission effect.

3.4. Influence of Relative Position of Transmitting and Receiving Coils on the Transmission Performance of MCRETS. Nondirectional propagation is a distinguishable feature of magnetically coupled resonance that sets it apart from the electromagnetic induction. In the electromagnetic induction coupling, a slight rotation and translation of the coil significantly induces energy loss in the receiving coil. However, when transmission is performed through MCRETS, the 


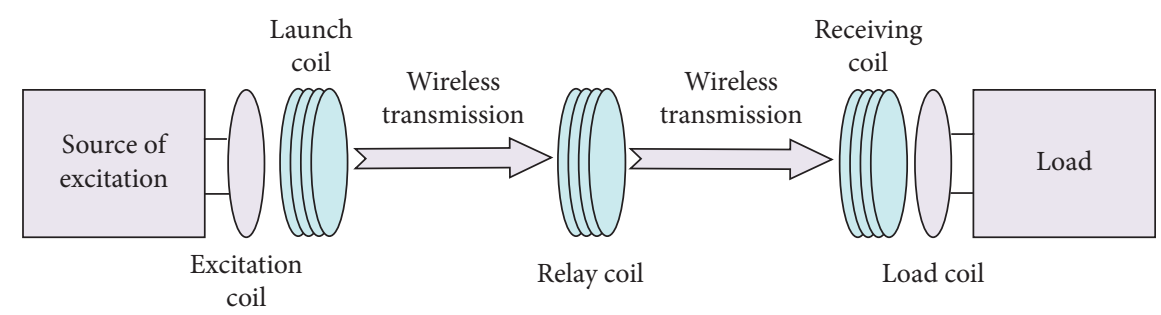

FIgURE 4: Schematic of the five-coil wireless energy transmission system.

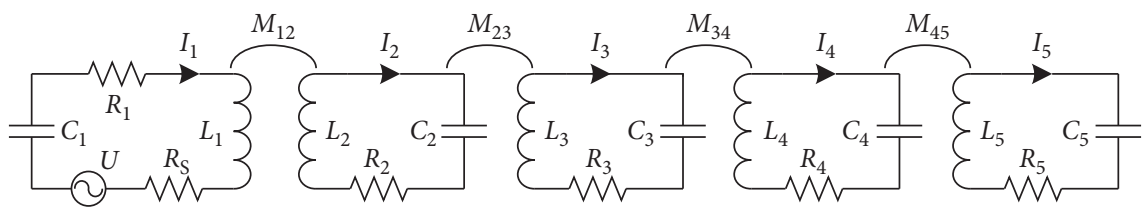

Figure 5: Equivalent circuit model for a five-coil wireless energy transfer system.

transmission and rotation of the coil within a certain transmission range will have a relatively small transmission loss. When the relative position of the transceiver coil goes beyond the range of the changing position, the rotation of the receiving coil has a considerable effect on the output power.

\section{Finite Element Method Verification}

In this paper, the launch coil and receiving coil are simulated by ANSYS 10.0 software. Because of the different analysis objects and different analysis methods, the nodal method of SOLID97 unit type is adopted in the analysis of the ray coil, which has the characteristics of simplicity, rapidity, and effectiveness. The simulation calculation of adding iron core between the launch coil and the receiving coil by using the edge method of the SOLID117 unit type can be simpler and more accurate.

Taking SOLID97 as an example, we set the degrees of freedom as AX, AY, and AZ. Then, the three-dimensional model of the cylindrical coil and the air can be built. Because the magnetic induction intensity produced by the coil model is relatively symmetrical, in order to save calculation time, the copper wire is chosen as the current-carrying winding coil, whose resistivity is $2.135 \times 10^{-8} \Omega \cdot m$ and relative permeability is 1 . The coil model is shown in Figure 6 . The coil diameter is $100 \mathrm{~mm}$, the height is $100 \mathrm{~mm}$, the wire diameter is $3 \mathrm{~mm}$, the number of turns is 5 , and the current per turn is $50 \mathrm{~A}$.

For the following simulation, the following assumptions and conventions are made: approximate material isotropy; the influence of temperature change is not considered for the time being; the air region is considered as infinite; when iron core is added to the simulation, the $\mathrm{B}-\mathrm{H}$ curve of iron core is approximately considered linear; when iron core is added to the simulation, the effect of eddy current demagnetization is not considered for the time being.

For the ANSYS analysis model, network partitioning is particularly important. The accuracy and speed of solution are greatly affected by the density of network partitioning.

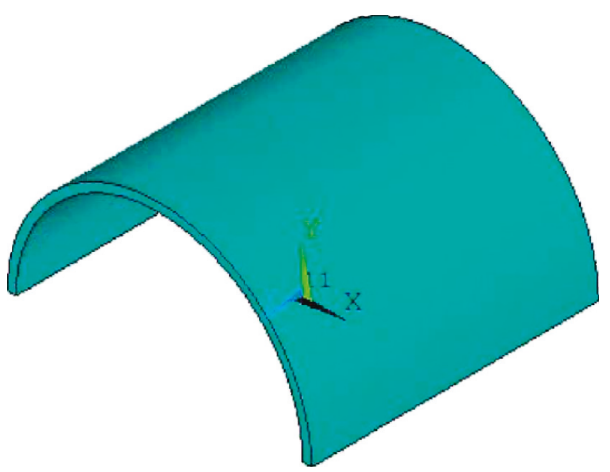

Figure 6: Cylindrical coil model.

In this paper, the coil and air dimensions of the model are quite different, so it cannot be divided in one time. The size of coil/iron core and air setting unit need to be separately divided freely. Considering the requirement of solving accuracy and solving time, the coil region needs to be partitioned in detail, while the air region follows the partitioning rule from inner to outer meshes to dense to sparse meshes. Figure 7 is a partition diagram with coils. Figure 8 is a general partition diagram of air and coils. The denser the black grid is, the finer the coil area is. The densest part in the middle is the coil.

The coil in simulation is a planar spiral coil. In order to effectively limit the number of subdivision units in FEM simulation, it is necessary to simplify the coil shape in modeling. Simplifying the coil shape will not affect the magnetic field distribution in the transmission channel too much. In the simulation software, the model is shown in Figure 9. The cross section is simplified, including the square cross section instead of the circular cross section of the conductor, whose side length is $5 \mathrm{~mm}$, the turn number is 8 turns, the maximum external diameter is $38 \mathrm{~cm}$, and the turn distance is $3 \mathrm{~mm}$.

During the simulation, Maxwell and Simplorer are used to simulate the magnetic field distribution. The result of magnetic field distribution is shown in Figure 10. The distribution of magnetic field intensity when the magnetic field 


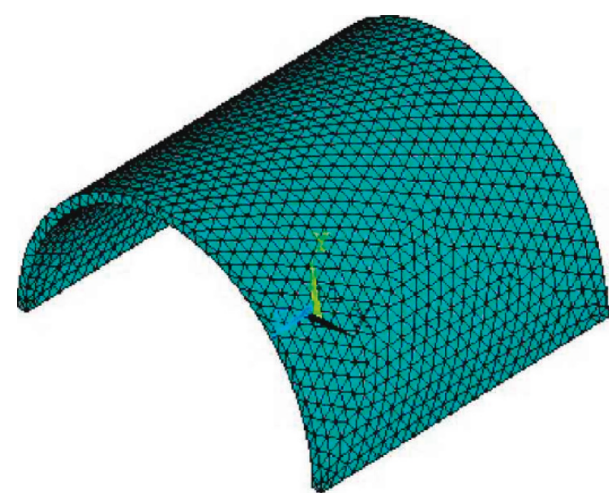

FIGURE 7: Grid of coils.

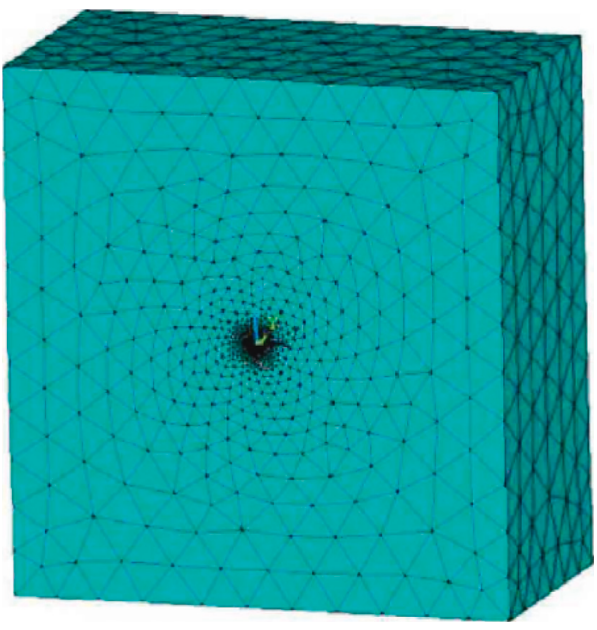

FIGURE 8: Overall grid with air.

intensity of the transmitting coil is maximum is shown in Figure 11. From the magnetic field intensity around the coil, it can be seen that the current is generated in the resonant coil at the receiving end.

Because of the similarity of the design simulation experiment process, this paper takes the above process as an example. By resetting the parameters of the simulation process, including transmission distance, system resonance frequency, relay structure, and relative position of transmitting and receiving coils, we can get multiple sets of simulation data through repeated simulation. In the next chapter, these simulated data will be used to compare and discuss with experimental data.

\section{Experimental Verification of the Transmission Performance of MCRETS}

The corresponding experimental physical device is built based on the working principle of MCRETS. The theoretical analysis results of the transmission performance of the wireless energy transmission system can be verified experimentally through this device, which is shown in Figure 12. The parameters of the relevant experimental equipment are shown in Table 1. The device comprises five parts, namely, transmitting device, transmitting coil, receiving coil, receiving device, and load. The transmitting device emits energy. The receiving coil receives energy and then powers the load. During the experiment, the input and output voltages, input and output currents, input and output powers, and other related data are displayed by the transmitter and receiver. Photographs of the receiving coil and the launch coil are presented in Figure 13, and the experimental setup is shown in Figure 14.

\subsection{Influence of Transmission Distance on the Transmission} Performance of MCRETS. The results of the transmission distance are experimentally verified. In the experiment, the position of the transmitting coil is kept constant, the receiving coil is moved to the right, and the transmission distance of the system is gradually increased. The relevant experimental data are collected by the transmitting and receiving devices. Data can be obtained from the theoretical analysis and experiment. The graph of the relationship between transmission distance and transmission power is shown in Figure 15(a) and that between transmission distance and transmission efficiency is shown in Figure 15(b).

From Figure 7, the output power of wireless energy transmission system initially increases and then decreases with the increase in transmission distance, whereas the transmission efficiency of the wireless energy transmission system decreases with the increase in transmission distance. These changes are consistent with the above theoretical analysis.

\subsection{Influence of Resonant Frequency on the Transmission} Performance of MCRETS. The resonant compensation capacitor of the wireless energy transmission system can be matched. The change in the resonant frequency of the system can be achieved by a change in the resonant compensation capacitor. In the experiment, the transmission distance is fixed at 2.5, 3.0, and $3.5 \mathrm{~cm}$. Experimental data are collected from the changes in the resonant frequency of the system and then graphed with simulated data to show the relationships between the resonant frequency and output power and between the resonant frequency and transmission efficiency. The graph of the relationship between the output power and the resonant frequency is shown in Figure 16(a) and that between the resonant frequency and transmission efficiency is shown in Figure 16(b).

From Figure 16, with transmission distance kept constant, the output power of the system initially increases and then decreases with the increase in the resonant frequency of the system, whereas transmission and resonant frequencies of the system have a direct relationship. However, the existence of the skin effect in the actual system will increase its transmission efficiency, and any increase in resonant frequency tends to be stable. Experimental and theoretical analyses of the transmission efficiency value gap show a gradual increase. Therefore, when the resonant frequency of the wireless energy transmission system is low, a large output power should be kept close to the transmission, whereas if the resonant frequency in the wireless energy transmission 


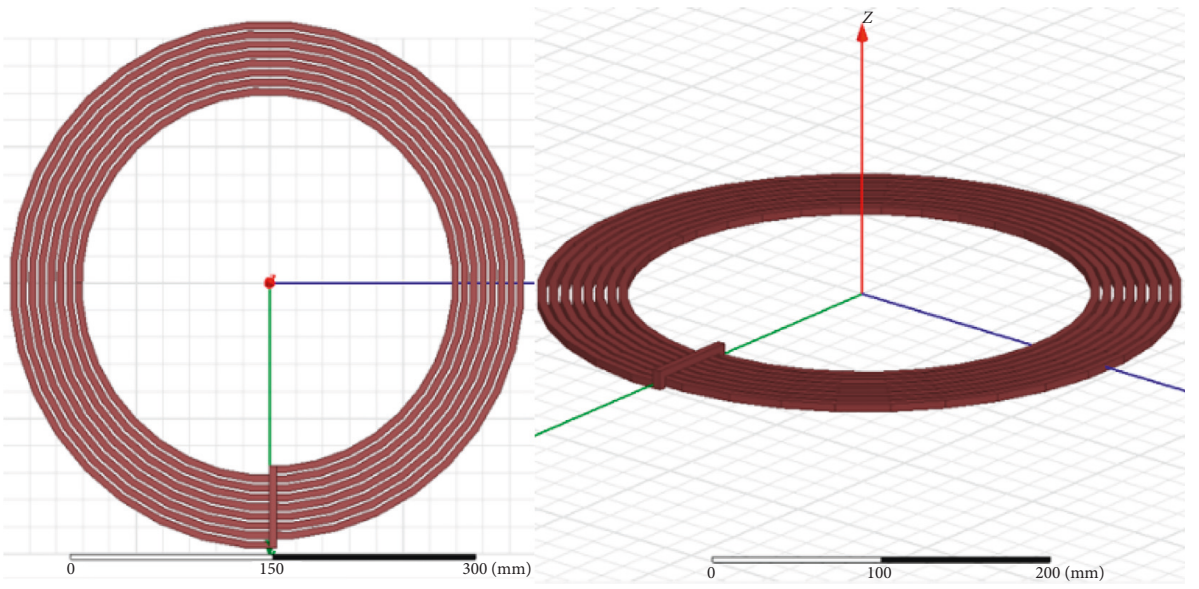

Figure 9: Coil 3D model drawn in Maxwell.

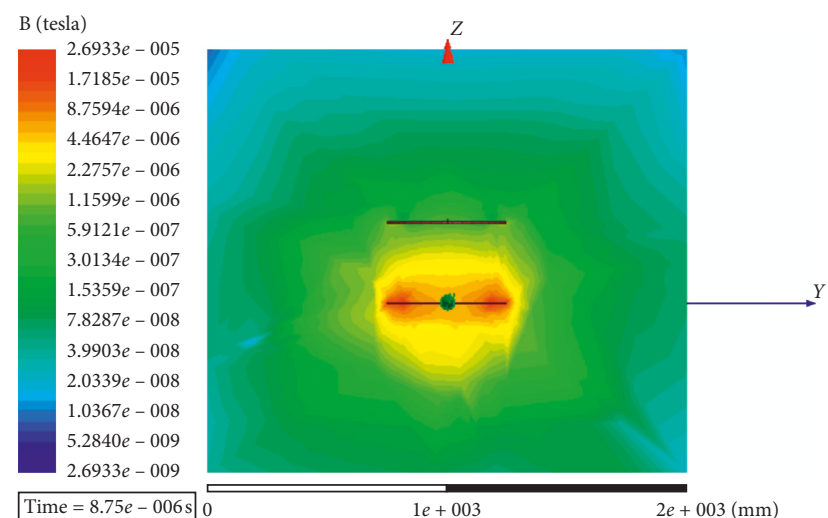

FIGURE 10: The maximum value of the magnetic field of the transmitting coil in transient field simulation.

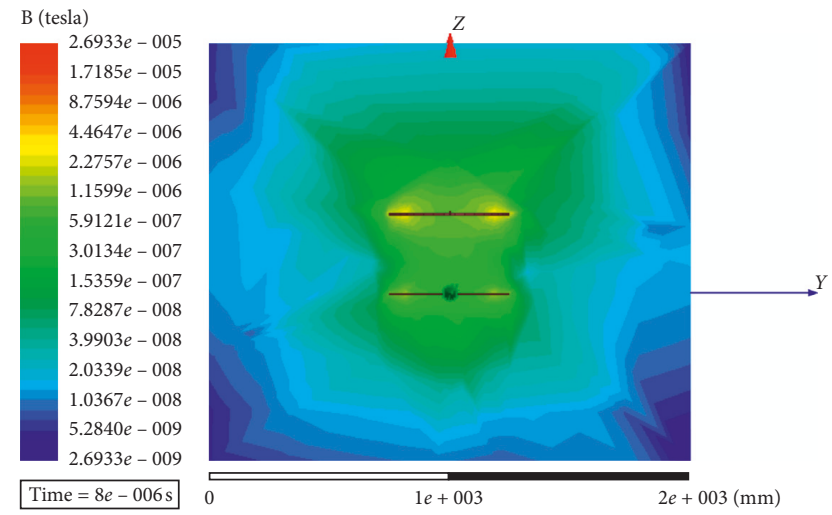

Figure 11: Transient field simulation receiver coil maximum magnetic field.

system is high, a large output power should be kept far from the transmission.

\subsection{Influence of Relay Coils on the Transmission Performance} of MCRETS. The experimental data of the relay and nonrelay coils are collected by the experimental devices. The

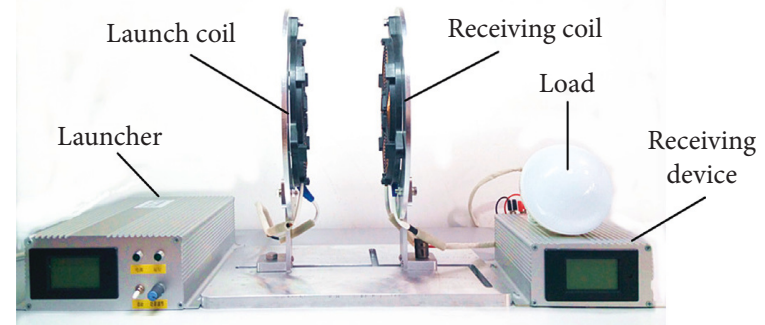

Figure 12: Physical diagram of MCRETS.

TABLE 1: Experimental equipment-related parameter.

\begin{tabular}{lc}
\hline Parameter item & Value \\
\hline Device input voltage & $220 \mathrm{~V} \mathrm{AC} / 50 \mathrm{~Hz}$ \\
Maximum device input & $1600 \mathrm{~W}, 220 \mathrm{~V} \mathrm{AC} /$ \\
& $8 \mathrm{~A}$ \\
Maximum output power of the device & $400 \mathrm{~W}, 24 \mathrm{~V} \mathrm{DC} /$ \\
Transmitting and receiving coil radius & $5 \mathrm{~A}$ \\
Transceiver turns & $3 \mathrm{~mm}$ \\
Coil winding radius & 20 \\
The minimum distance between & $100 \mathrm{~mm}$ \\
transmitting and receiving coils & $10 \mathrm{~mm}$ \\
\hline
\end{tabular}

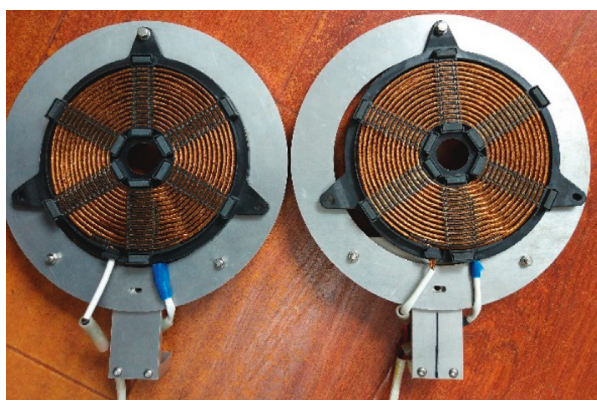

FIGURE 13: Receiving coil and launch coil of the practical MCRETS system. 


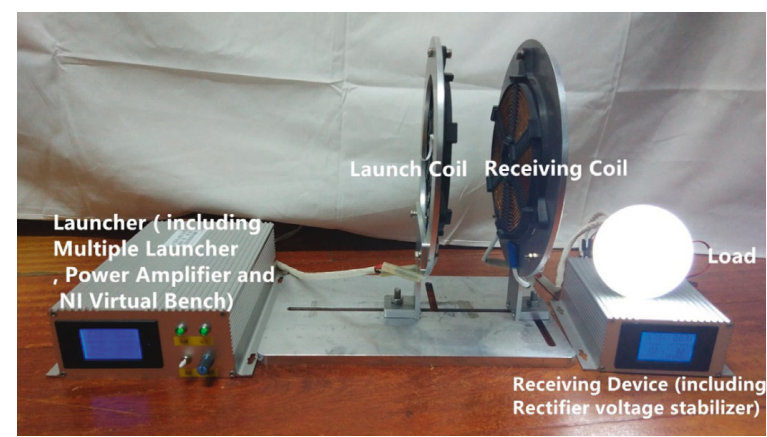

Figure 14: Experimental setup of the proposed MCRETS system.

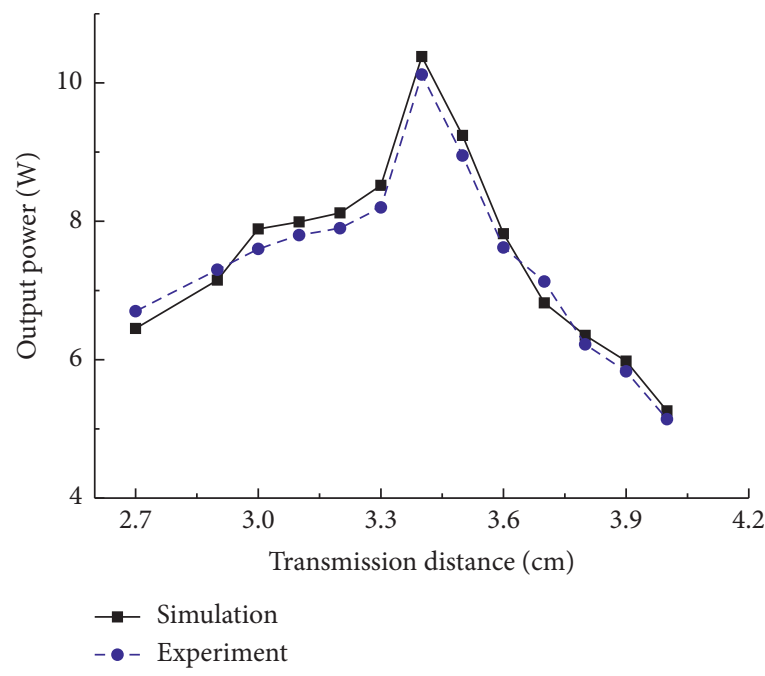

(a)

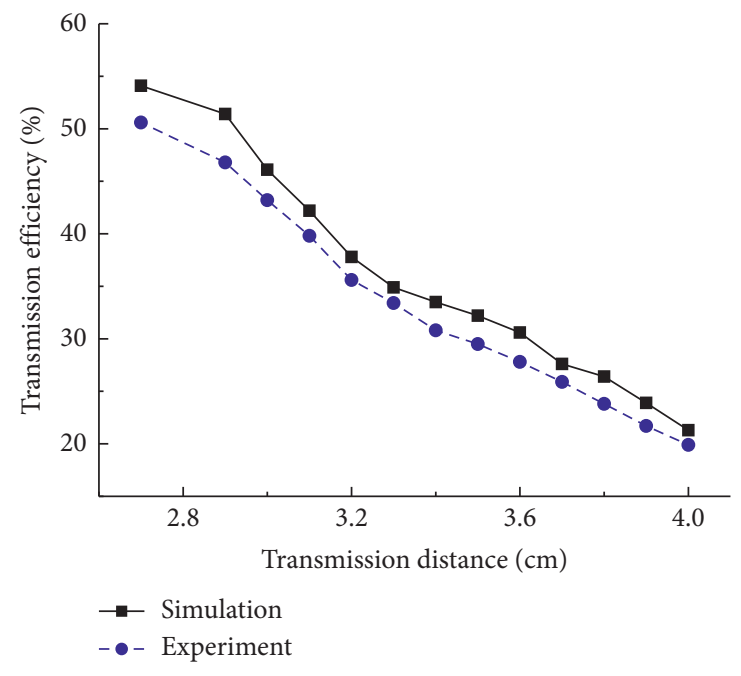

(b)

Figure 15: Transmission distance-output power and transmission distance-transmission efficiency curves.

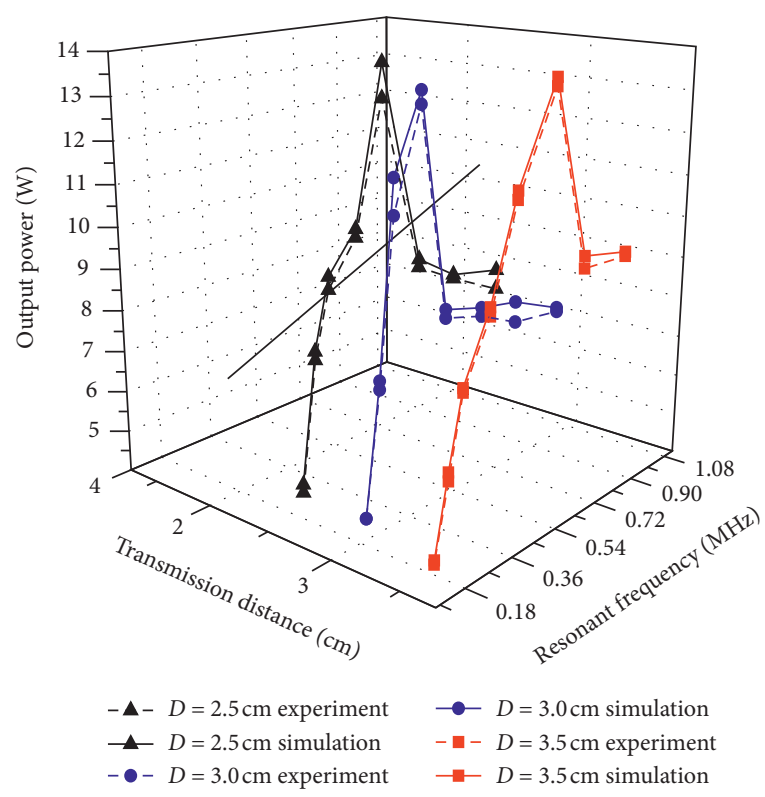

(a)

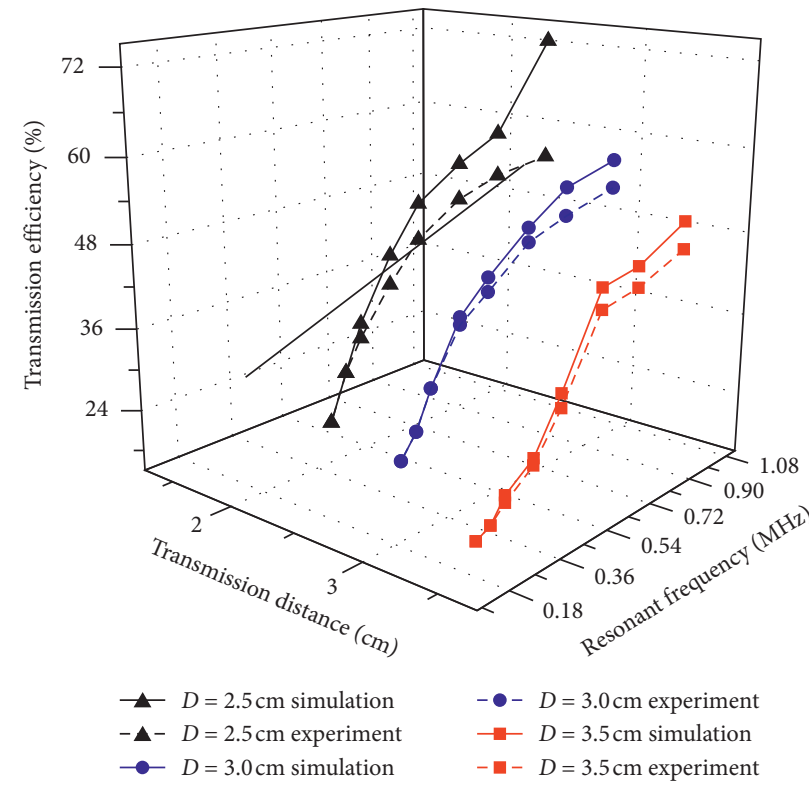

(b)

FIGURE 16: (a) Resonant frequency-output power efficiency relationships; (b) resonant frequency-transmission efficiency relationships. 
relevant experimental data, which are fluctuations in the receiving terminal voltage as transmission distance changes, are collected, and the curves of the voltage and transmission distance of the relay and the nonrelay are drawn. The comparison chart is shown in Figure 17.

The variation curve in Figure 17 reveals that the voltage value of the receiver terminal is inversely proportional to the transmission distance. The curve for the transmission distance of the relay coil is slower than the curve for the transmission distance of the nonrelay coil. The magnitude of the attenuation of the magnetic field or the relay coil increases the range of the magnetic field. At the same time, the relay coil effectively increases the transmission distance of the system and enhances the transmission effect. The experimental results are consistent with the theoretical analysis results.

5.4. Influence of the Relative Position of Receiving and Transmitting Coils on the Transmission Performance of MCRETS. Findings from the theoretical analysis in this study are verified through an experiment. Before the start of the experiment, the angle between the receiving coils is set at $-40^{\circ}$ and the receiving coil is then rotated slowly in the direction vertical to the coil diameter until it rotates to an angle of $35^{\circ}$. The value of the output power during rotation is recorded. The output power varies with the rotation angle of the receiving coil. The experimental data are graphed in Figure 18. However, the theoretical derivation of the relative position of the launch and receiving coils has not yet been accurate. We only conducted preliminary verification through experiments and obtained preliminary conclusions after verification. In the future research, we will further derive the theoretical basis.

5.5. Transmission Characteristics of SS Topological System in Maximum Power Product. The above theoretical analysis and experiments reveal that when the system output power is at maximum, the transmission efficiency is often extremely low. A wireless energy transmission system is a relatively easily affected system, and its transmission performance is easily affected by many factors. At present, many scholars are analyzing the power and efficiency of the wireless energy transmission system, but this cannot guarantee the optimal transmission performance of the system. This paper combines the power and efficiency problems of wireless energy system transmission, that is, taking into account the power and efficiency of transmission and optimizing the system transmission performance. This kind of research is still rare.

The literature [28] compared and analyzed the two models of the four-coil end-opening coil and the shortcircuited string-capacitor four-coil based on the spiral coil through the electromagnetic simulation. But this is only from the point of view of electromagnetic simulation, lack of specific reasoning calculation process cannot optimize the output power and transmission efficiency of the system, and it is even more impossible to optimize the system

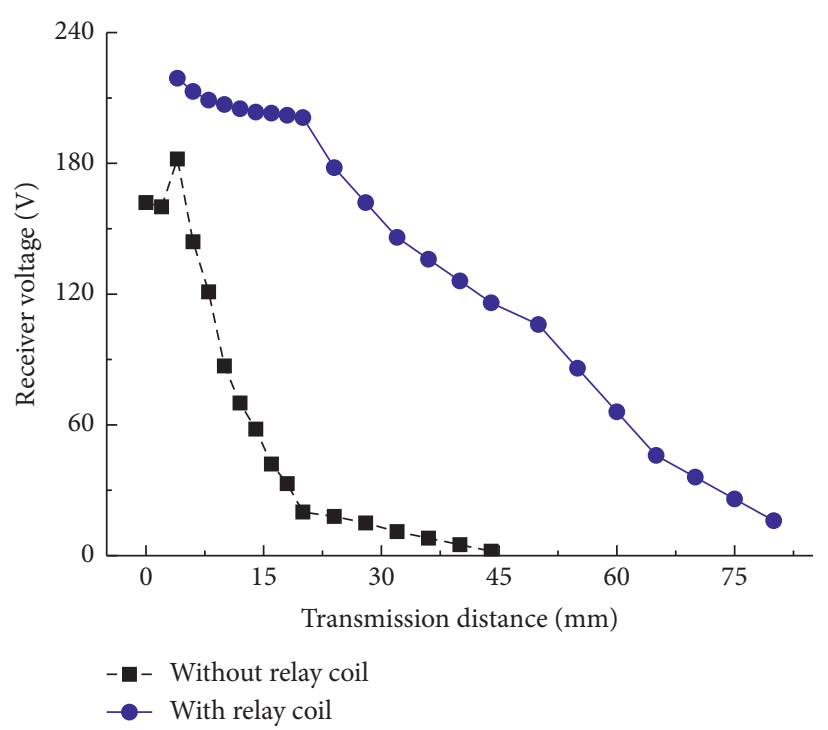

FIGURE 17: Receipt of voltage change of the experimental device, with distance changing with or without relay coil.

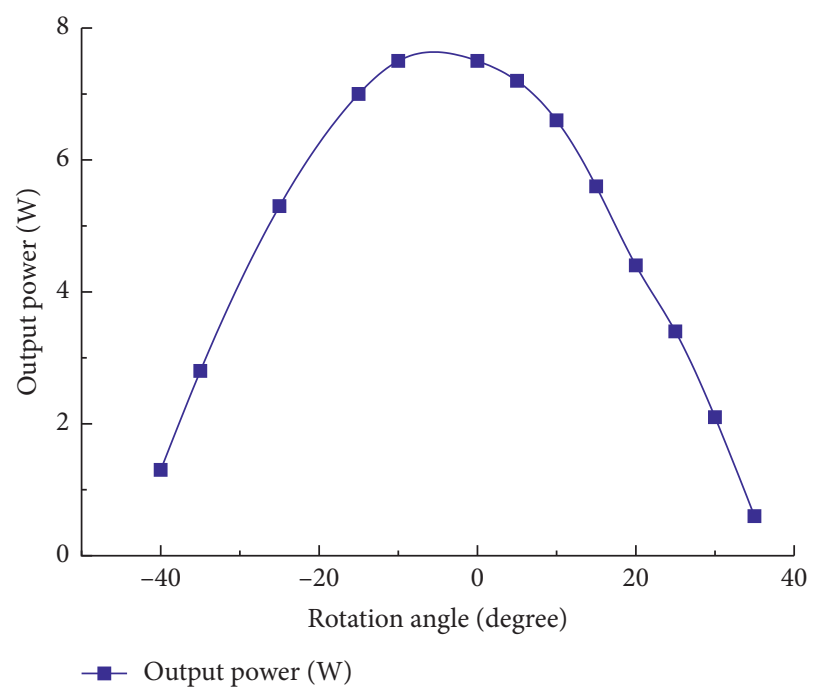

FIGURE 18: Output voltage-direction curve.

transmission performance. In this literature [29], two basic structures are analyzed and the equations of the transfer efficiency are deduced. Two important factors, namely, the transfer quality factor and the load matching factor, are explored. However, there is no research on how to balance the transmission efficiency and power in order to optimize the transmission performance of the wireless energy transmission system.

For the above problem, this study proposes the power product. The optimal parameters for the wireless energy transmission system are analyzed to allow higher transmission efficiency in the system even when the output power is high. The power product is the product of the output power and the transmission efficiency, and it can be expressed by $\Psi$ (its unit is the product of two variable units: $W$ ), as shown in the following: 


$$
\Psi=P_{0} \cdot \eta=\frac{(\omega M)^{4} U_{i}^{2} R_{\mathrm{L}}}{\left[(\omega M)^{2}+\left(R+R_{0}\right)\left(R_{0}+R_{\mathrm{L}}\right)\right]^{3}} .
$$

For simplicity, the wireless energy transmission system is assumed to be in the ideal state and that the remaining items are constant; thus, the amount of change is only one-unit resonance frequency. It is necessary to set a series of parameters according to the idealized situation. According to the parameters set, the related formulas can be analyzed more effectively and conveniently. The analysis is simpler and clearer. System parameters are listed in Table 2. From equation (15), the curve of the power product $\Psi$ and resonant frequency $\omega$ can be obtained, as shown in Figure 19. In order to find a relatively optimal value, it can meet the transmission power and transmission efficiency in a certain situation to achieve a relatively optimal value.

In Figure 11, when at the peak where $d \Psi / d \omega=0$, the extreme point can be derived as follows:

$$
\omega_{0}=\frac{\sqrt{2\left(R+R_{0}\right)\left(R_{0}+R_{\mathrm{L}}\right)}}{M} .
$$

The solvable output power $P_{0-\Psi}$ and transmission efficiency $\eta_{\Psi}$ of the system are follows:

$$
\begin{aligned}
P_{0-\Psi} & =\frac{2 U_{i}^{2}}{9 R_{W}}, \\
\eta_{\Psi} & =\frac{2}{3} .
\end{aligned}
$$

From the above analysis, it shows that when $\omega=\sqrt{\left(R+R_{0}\right)\left(R_{0}+R_{W}\right)} / M$, the output power reaches the optimal value, and we can get the corresponding output power $P_{0-P}$ and the transmission efficiency at the maximum output power $\eta_{P}$ :

$$
\begin{aligned}
P_{0-P} & =\frac{U_{i}^{2}}{4 R_{W}}, \\
\eta_{P} & =\frac{1}{2} .
\end{aligned}
$$

$\alpha$ and $\beta$ are the change ranges of the output power and transmission efficiency, respectively. when $\alpha=-11.1 \%$ and $\beta=33.3 \%$, the output power of the system is reduced by $11.1 \%$, and the transmission efficiency is increased by $33.3 \%$.

Similarly, if other conditions are not changed, only the change in transmission distance is controlled. When the transmission distance satisfies $D^{2}=\sqrt[3]{\omega^{2} T^{2} / 2\left(R+R_{0}\right)\left(R_{0}+R_{W}\right)}$ $-2 r^{2}$, the system's power product reaches its maximum. At this time, the output power is $P_{0-\psi}=2 U_{i}^{2} / 9 R_{W}$, and the transmission efficiency is $\eta_{\psi}=2 / 3$. Under the maximum power index, when the transmission distance satisfies $D^{2}=$ $\sqrt[3]{\omega^{2} T^{2} / 2\left(R+R_{0}\right)\left(R_{0}+R_{W}\right)}-2 r^{2}$, the output power takes the maximum value $P_{0-P}=U_{i}^{2} / 4 R_{W}$, and the transmission efficiency is then $\eta_{P}=0.5$.

In summary, the use of power product parameters for optimization ensures that the output and transmission
TABLE 2: System parameters.

\begin{tabular}{lcc}
\hline DC input voltage & LS $(\mathrm{Ld})$ & $R_{0}$ \\
\hline $15 \mathrm{~V}$ & $14.25 \mu \mathrm{H}$ & $0.27 \Omega$ \\
$\mathrm{F}$ & $\mathrm{RW}$ & $\mathrm{R}$ \\
$500 \mathrm{kHz}$ & $5 \Omega$ & $2.5 \Omega$ \\
\hline
\end{tabular}

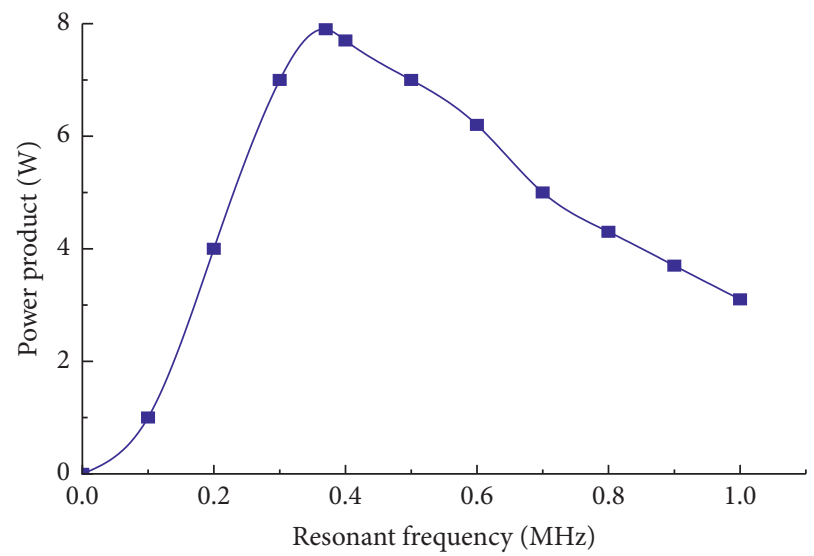

FIGURE 19: Relationship between the power product and resonant frequency.

powers will be relatively high, which optimizes the transmission performance.

\section{Conclusions}

This study introduced the MCRETS model, obtaining an optimal topology through modeling and analysis of its four topological structures. Theoretical analysis and experimental verification of the factors, which affect the transmission performance of the MCRETS (i.e., transmission distance, resonant frequency, relay coil, and relative position of transmitting and receiving coils) were conducted and discussed. The theoretical analysis and experimental results show that these factors greatly influence the transmission performance of the wireless energy transmission system, such that the factors can further improve the transmission performance once optimized. Based on the above analysis, this study proposed the parameters of the power product to ensure a relatively high transmission power with the maximized performance. This concept is of great significance in practical application. For example, the problem of choosing the highest power or higher charging efficiency has always existed. With the concept of power product, the maximum efficiency product can be preferentially selected, which is more conducive to the optimization of charging performance.

However, the research on the transmission structure of MCRETS is limited because it is based on a two-coil structure. Further research will study the effect of single or multiple power supply charging using a one-to-one structure or a network of structures. Based on the analysis, further research on the design for a more efficient, more powerful, and more stable MCRETS is also desirable. 


\section{Data Availability}

The data used to support the findings of this study are available from the corresponding author upon request.

\section{Conflicts of Interest}

The authors declare that they have no conflicts of interest.

\section{Acknowledgments}

The authors would like to acknowledge the support from the National Key R\&D Program of China (grant no. 2018YFB1304600), CAS Interdisciplinary Innovation Team (grant no. JCTD-2018-11), DREAM project of EU FP7-ICT (grant no. 611391), and National Natural Science Foundation of China (grant nos. 51575412, 51575338, and $5157540)$.

\section{References}

[1] S. Y. R. Hui, W. Zhong, and C. K. Lee, "A critical review of recent progress in mid-range wireless power transfer," IEEE Transactions on Power Electronics, vol. 29, no. 9, pp. 45004511, 2014.

[2] J. G. Kim, G. Wei, M. H. Kim, J. Y. Jong, and C. Zhu, “A comprehensive study on composite resonant circuit-based wireless power transfer systems," IEEE Transactions on Industrial Electronics, vol. 65, no. 6, pp. 4670-4680, 2017.

[3] A. Ibrahim, M. Meng, and M. Kiani, "A comprehensive comparative study on inductive and ultrasonic wireless power transmission to biomedical implants," IEEE Sensors Journal, vol. 18, no. 9, pp. 3813-3826, 2018.

[4] M. Soljacic, Wireless Energy Transfer Can Potentially Recharge Laptops, Cell Phones without Cords, Institute of Technology, San Francisco, MA, USA, 2006.

[5] K. M. Silay, C. Dehollain, and M. Declercq, "Inductive power link for a wireless cortical implant with biocompatible packaging," in Proceedings of the Sensors, pp. 94-98, Kona, HI, USA, November 2010.

[6] B. Zhu, J. Li, W. Hu, and X. Gao, "Review of magnetic coupling resonance wireless energy transmission," International Journal of $u$ - and e-Service, Science and Technology, vol. 8, no. 3, pp. 257-272, 2015.

[7] T. Deyle and M. Reynolds, "Surface based wireless power transmission and bidirectional communication for autonomous robot swarms," in Proceedings of the IEEE International Conference on Robotics and Automation, pp. 1036-1041, Pasadena, CA, USA, June 2008.

[8] F. Zhang, S. Hackworth, X. Liu et al., "Wireless power delivery for wearable sensors and implants in body sensor networks," in Proceedings of the Engineering in medicine and biology society, pp. 692-695, Buenos Aires, Argentina, August 2010.

[9] Y. Jang and M. M. Jovanovic, "A contactless electrical energy transmission system for portable-telephone battery chargers," IEEE Transactions on Industrial Electronics, vol. 50, no. 3, pp. 257-272, 2003.

[10] R. Johari, J. V. Krogmeier, and D. J. Love, "Analysis and practical considerations in implementing multiple transmitters for wireless power transfer via coupled magnetic resonance," IEEE Transactions on Industrial Electronics, vol. 61, no. 4, pp. 1774-1783, 2014.
[11] M. Pinuela, D. C. Yates, S. Lucyszyn, and P. D. Mitcheson, "Maximizing DC-to-load efficiency for inductive power transfer," IEEE Transactions on Power Electronics, vol. 28, no. 5, pp. 2437-2447, 2013.

[12] M. Kiant, U. M. Jow, and M. Ghovanloo, "Design and optimization of a 3-coil inductive link for efficient wireless power transmission," IEEE Transactions on Biomedical Circuits and Systems, vol. 5, no. 6, pp. 579-591, 2011.

[13] T. Sun, X. Xie, G. Li et al., "A two-hop wireless power transfer system with an efficiency-enhanced power receiver for motion-free capsule endoscopy inspection," IEEE Transactions on Biomedical Engineering, vol. 59, no. 11, pp. 3247-3254, 2012.

[14] C. Jiang, K. T. Chau, C. Liu, and C. H. T. Lee, "An overview of resonant circuits for wireless power transfer," Energies, vol. 10, no. 7, p. 894, 2017.

[15] A. Kurs, A. Karalis, R. Moffatt, J. D. Joannopoulos, P. Fisher, and M. Soljacic, "Wireless power transfer via strongly coupled magnetic resonances," Science, vol. 317, no. 5843, pp. 83-86, 2007.

[16] K. Jinwook, S. Hyeon-Chang, K. Kwan-Ho et al., "Efficiency analysis of magnetic resonance wireless power transfer with intermediate resonant coil," IEEE Antennas and Wireless Propagation Letters, vol. 10, pp. 389-392, 2011.

[17] A. K. Ramrakhyani, S. Mirabbasi, and M. Chiao, "Design and optimization of resonance-based efficient wireless power delivery systems for biomedical implants," IEEE Transactions on Biomedical Circuits and Systems, vol. 5, no. 1, pp. 48-63, 2011.

[18] X. Zhang, S. L. Ho, and W. N. Fu, "Analysis and optimization of magnetically coupled resonators for wireless power transfer," IEEE Transactions on Magnetics, vol. 48, no. 11, pp. 4511-4514, 2012.

[19] M. Sawan, Y. Yamu Hu, and J. Coulombe, "Wireless smart implants dedicated to multichannel monitoring and microstimulation," IEEE Circuits and Systems Magazine, vol. 5, no. 1, pp. 21-39, 2005.

[20] D. Ahn and S. Hong, "A study on magnetic field repeater in wireless power transfer," IEEE Transactions on Industrial Electronics, vol. 60, no. 1, pp. 360-371, 2013.

[21] F. Zhang, S. Hackworth, X. Liu et al., "Wireless energ y transfer platform for medical sensors and implantable devices," in Proceedings of the 2009 Annual International Conference of the IEEE Engineering in Medicine and Biology Society, pp. 1045-1048, Minneapolis, MN, USA, September 2009.

[22] D. Ahn, S. M. Kim, S. W. Kim, J. I. Moon, and I. K. Cho, "Wireless power transfer receiver with adjustable coil output voltage for multiple receivers application," IEEE Transactions on Industrial Electronics, vol. 66, no. 5, pp. 4003-4012, 2018.

[23] D. H. Kim and D. Ahn, "Maximum efficiency point tracking for multiple-transmitters wireless power transfer," IEEE Transactions on Power Electronics, 2019.

[24] K. M. Silay, C. Dehollain, and M. Declercq, "Inductive power link for a wireless cortical implant with two-body packaging," IEEE Sensors Journal, vol. 11, no. 11, pp. 2825-2833, 2011.

[25] M. K. Watfa, H. AlHassanieh, and S. Selman, "Multi-hop wireless energy transfer in WSNs," IEEE Communications Letters, vol. 15, no. 12, pp. 1275-1277, 2011.

[26] E. Waffenschmidt and T. Staring, "Limitation of inductive power transfer for consumer applications," in Proceedings of the 2009 13th European Conference on Power Electronics and Applications, pp. 1-10, IEEE, Barcelona, Spain, September 2009. 
[27] J. O. Mur-Miranda, G. Fanti, Y. Feng et al., "Wireless power transfer using weakly coupled magnetostatic resonators," in Proceedings of the IEEE ECCE, pp. 4179-4186, Atlanta, GA, USA, September 2010.

[28] H. H. T. AmanoN Kikuma et al., "A consideration of open and short-end type helical antennas for magnetic-coupled resonant wireless power transfer," in Proceedings of the 2012 6th European Conference on Antennas and Propagation (EUCAP), pp. 3009-3013, Prague, Czech Republic, March 2012.

[29] Y. Zhang, Z. Zhao, and K. Chen, "Frequency decrease analysis of resonant wireless power transfer," IEEE Transactions on Power Electronics, vol. 29, no. 3, pp. 1058-1063, 2014. 


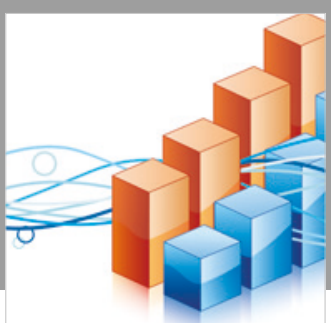

Advances in

Operations Research

\section{-n-m}
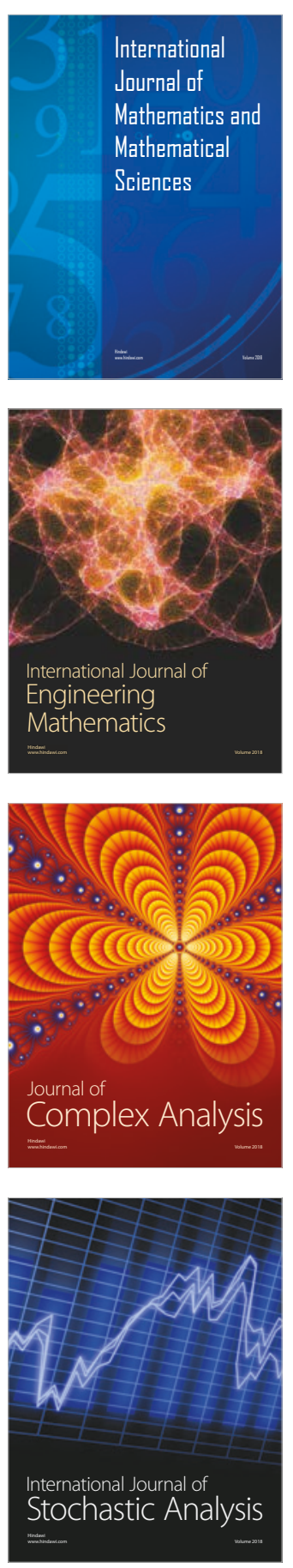
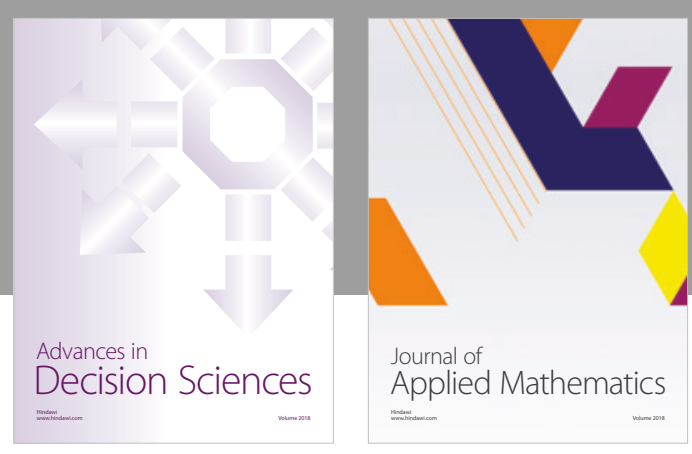

Journal of

Applied Mathematics
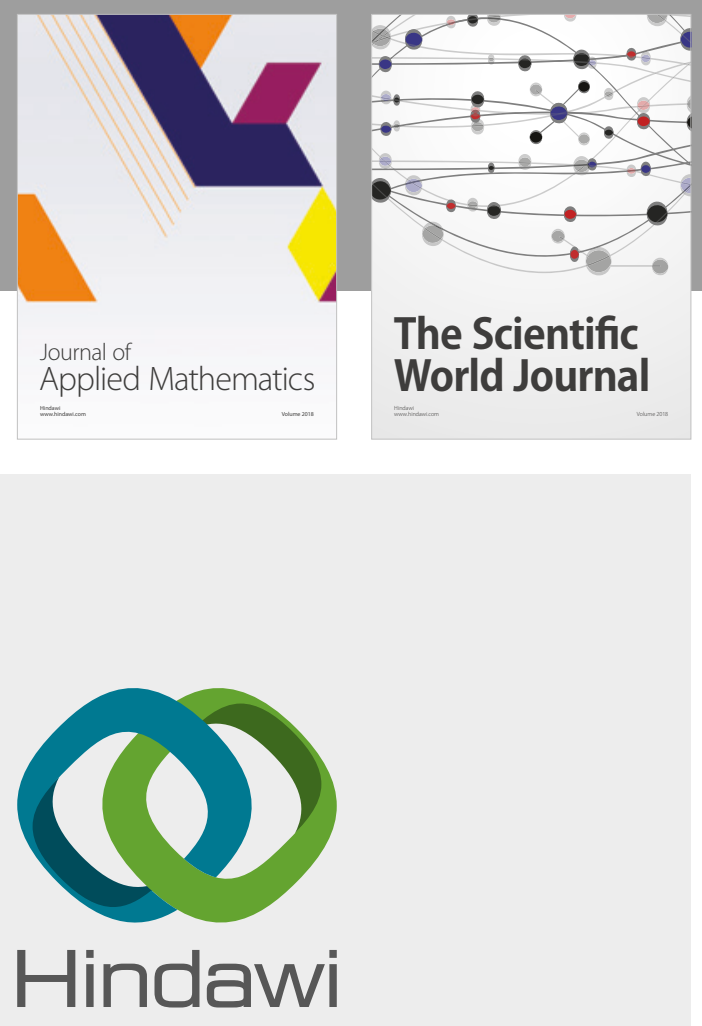

Submit your manuscripts at

www.hindawi.com

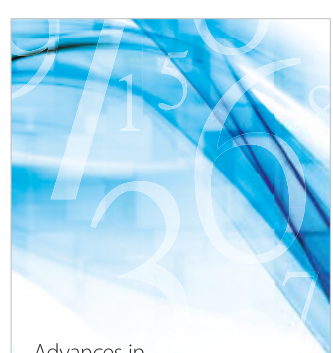

Advances in
Numerical Analysis
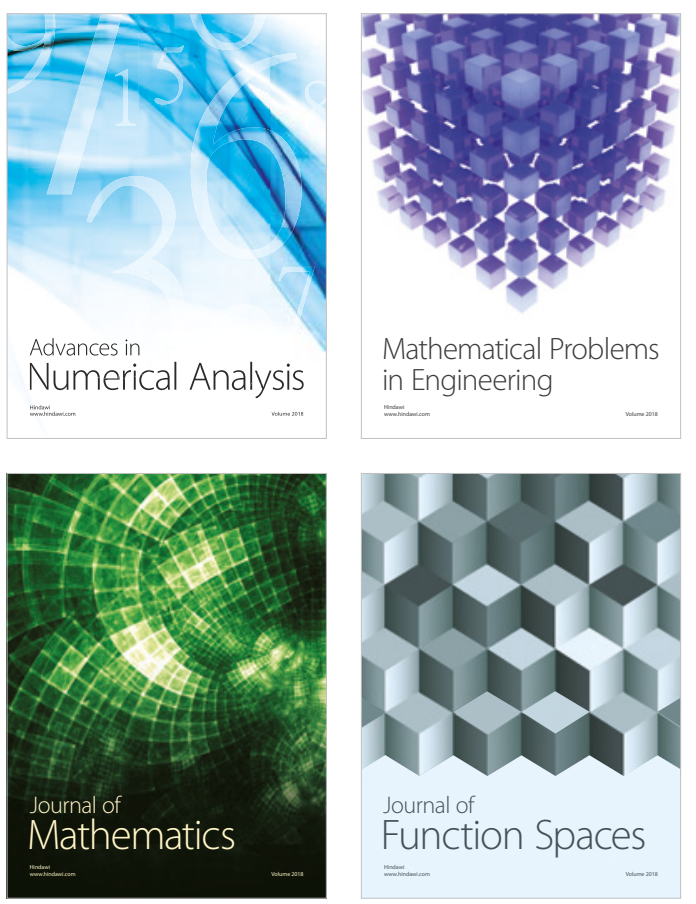

Mathematical Problems in Engineering

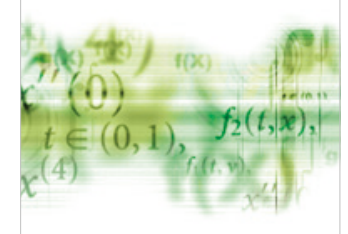

International Journal of

Differential Equations

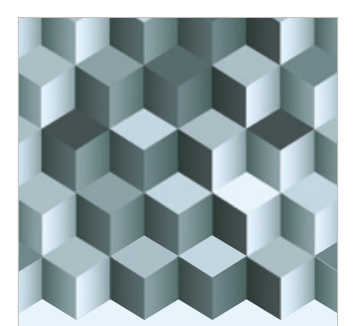

Journal of

Function Spaces
The Scientific

World Journal

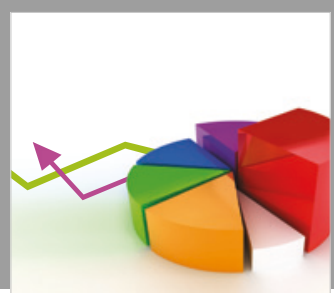

Journal of

Probability and Statistics
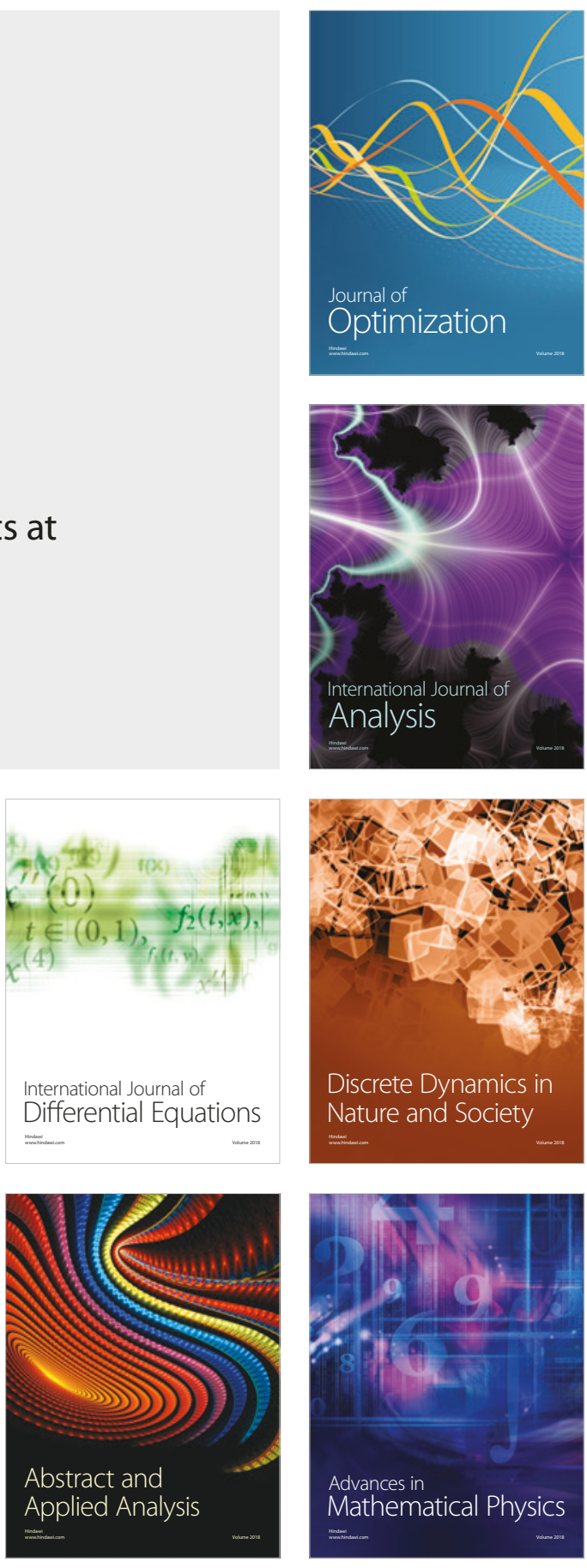